\title{
Endoscopic third ventriculostomy and choroid plexus cauterization with a rigid neuroendoscope in infants with hydrocephalus
}

\author{
Alexander G. Weil, MD, FRCSC, Aria Fallah, MD, MSc, FRCSC, Parthasarathi Chamiraju, MD, \\ John Ragheb, MD, and Sanjiv Bhatia, MD \\ Division of Pediatric Neurosurgery, Department of Neurosurgery, University of Miami Miller School of Medicine, Miami Children's \\ Hospital, Miami, Florida
}

\begin{abstract}
OBJECTIVE Combining endoscopic third ventriculostomy (ETV) with choroid plexus cauterization (CPC) has been shown to improve the success rate compared with ETV alone in infants (less than 24 months) with hydrocephalus who were treated in developing countries. The authors sought to evaluate the safety and efficacy of this procedure, using a rigid neuroendoscope, in a single North American center, and to assess whether the ETV success score (ETVSS), the CURE Children's Hospital of Uganda ETVSS (CCHU ETVSS), and other pre- and intraoperative variables could predict success.
\end{abstract}

METHODS The authors performed a retrospective review of consecutive ETV/CPC procedures performed using a rigid neuroendoscope to treat infantile hydrocephalus. The infants underwent the procedure at Miami Children's Hospital between January 2007 and 2014, with at least one postoperative follow-up. Duration of follow-up or time to failure of ETVI $\mathrm{CPC}$, the primary outcome measure, was documented. A repeat CSF diversion procedure or death was considered as a failure of ETVICPC. The time to event was measured using a Kaplan-Meier analysis. The authors analyzed ETVSS, CCHU ETVSS, and pre- and intraoperative variables to determine their suitability to predict success.

RESULTS Eighty-five patients ( 45 boys) with a mean age of 4.3 months (range 1 day to 20 months) underwent ETVI CPC. Etiology included intraventricular hemorrhage of prematurity in 44 patients (51.7\%), myelomeningocele (MMC) in $7(8.2 \%)$, congenital aqueductal stenosis in $12(14.1 \%)$, congenital communicating hydrocephalus in $6(7.1 \%)$, DandyWalker complex in $6(7.1 \%)$, postinfectious hydrocephalus in $5(5.8 \%)$, and other cause in $5(5.8 \%)$. Six procedure-related complications occurred in $5(5.8 \%)$ patients, including 2 hygromas, 1 CSF leak, and 3 infections. There were 3 mortalities in this cohort. ETVICPC was successful in $42.1 \%, 37.7 \%$, and $36.8 \%$ of patients at 6,12 , and 24 months follow-up, respectively. The median $(95 \% \mathrm{Cl})$ time to ETV/CPC failure was 4.0 months (0.9-7.1 months). In univariate analyses, both the ETVSS (hazard ratio [HR] 1.03; 95\% Cl 1.01-1.05; $p=0.004$ ) and CCHU ETVSS (HR 1.48; 95\% Cl 1.04-2.09; $p=.028$ ) were predictive of outcome following ETVICPC. In multivariate analysis, the presence of prepontine scarring was associated with ETV/CPC failure ( $\mathrm{HR} 0.34 ; 95 \% \mathrm{Cl} 0.19-0.63 ; p<0.001)$. Other variables, such as radiological criteria (prepontine interval, prepontine space, aqueductal stenosis, Third Ventricular Morphology Index) and intraoperative findings (ventriculostomy pulsations, extent of $\mathrm{CPC}$ ), did not predict outcome.

CONCLUSIONS ETVICPC is a feasible alternative to ETV and ventriculoperitoneal shunt in infants with hydrocephalus. Both the ETVSS and CCHU ETVSS predicted success following ETVICPC in this single-center North American cohort of patients.

http://thejns.org/doi/abs/10.3171/2015.5.PEDS14692

KEY WORDS hydrocephalus; endoscopic third ventriculostomy; choroid plexus 
$\mathrm{E}$ NDOSCOPIC third ventriculostomy (ETV) is the treatment of choice for obstructive hydrocephalus in children. ${ }^{8,26}$ However, success rates drop significantly in certain populations, notably infants, patients with a history of prior hemorrhage or infection, or that have previously harbored a ventriculoperitoneal shunt (VPS). ${ }^{4,9,11,18,21,24}$ The ETV success score (ETVSS) has been developed and validated to predict ETV success based on these variables. ${ }^{24}$ There is strong evidence in sub-Saharan Africa that the addition of choroid plexus cauterization (CPC) to ETV can double success rates in hydrocephalus related to aqueductal stenosis (AS), myelomeningocele (MMC), or Dandy-Walker complex (DWC) and triple success rates in infants with postinfectious or communicating hydrocephalus. ${ }^{29,46,49,51,55}$ The CURE Children's Hospital of Uganda ETVSS (CCHU ETVSS), a scoring system that accounts for the extent of CPC, has since been developed and applied to this patient population. ${ }^{53}$

However, the socioeconomic milieu, health-care system, etiology, and natural history of hydrocephalus are different between developing and developed countries. ${ }^{30,53}$ It is unclear whether the documented safety and effectiveness of adding CPC to ETV in sub-Saharan Africa applies to infants with hydrocephalus in developed countries. ${ }^{5,29,44,50}$ Furthermore, it is unclear which infants are ideal candidates for this procedure, as the applicability of the ETVSS and CCHU ETVSS and other putative predictors of success have not been studied extensively in North American infants with hydrocephalus. ${ }^{29}$

We report a single-center, North American experience with ETV/CPC for infantile hydrocephalus. We sought to evaluate the feasibility, safety, and efficacy of ETV/CPC in this mixed cohort of North American infants with hydrocephalus. Our secondary objective was to assess whether the ETVSS and CCHU ETVSS could predict success following ETV/CPC in this cohort of patients. Finally, in an effort to identify which patients are candidates for ETV/ $\mathrm{CPC}$, we evaluated whether other preoperative clinical, radiological, or intraoperative variables could predict ETV/ CPC success.

\section{Methods}

\section{Patient Population}

After obtaining institutional ethics board approval, we identified all ETV/CPC procedures performed at Miami Children's Hospital and Jackson Memorial Hospital between July 2007 and May 2014. All infants and young toddlers (aged less than 24 months at the time of surgery) with at least one follow-up were included in the study. For simplicity, this group of patients (age less than 24 months) will be defined as infants in this study.

\section{Patient Selection}

There was no standard protocol for patient selection for ETV/CPC or other techniques (ETV, CSF shunting) during the study period. Selection of patients for ETV/CPC was left to the discretion of the treating neurosurgeon. In general, ETV/CPC was considered as a potential first-line treatment for patients with hydrocephalus and known risk factors of failure following ETV, such as younger age or a history of infection or intraventricular hemorrhage (IVH).

\section{Surgical Technique}

The surgical technique for ETV/CPC has been described in detail elsewhere (Fig. 1). ${ }^{5}$ Briefly, the technique involves performing an ETV through a frontal bur hole followed by ipsilateral CPC, septostomy, and contralateral CPC, cauterizing the choroid plexus along the choroidal fissure from the foramen of Monro to the posterior temporal horn using a rigid neuroendoscope (Storz Oi [Karl Storz GmbH \& Co. KG], Aesculap, Inc., or Claris [Medtronic]) (Fig. 2). The extent of CPC varied depending on ventricle size and septal anatomy.

\section{Data Collection}

We collected several preoperative, intraoperative, and postoperative variables through medical chart review to identify potential predictors of failure. Preoperative variables included patient age, etiology of hydrocephalus, presence of a prior CSF reservoir, prior external ventricular drain or VPS, prior CSF infection, and preoperative imaging ventricular variables, including the fronto-occipital horn ratio (FOHR), ${ }^{22,23,33}$ third ventricle morphology (lamina terminalis and/or tuber cinereum bulging) and Third Ventricular Morphology Index (TVMI), ${ }^{12}$ third ventricle width (TVW), prepontine interval (PPI), ${ }^{42}$ prepontine space (PPS; distance from midpons to clivus), 5 and presence/absence of AS (Fig. 3). ${ }^{5}$

Intraoperative variables were obtained from operative reports and included year of surgery, type of neuroendoscope, details of ETV (prepontine scarring prior to ETV, pulsation of third ventricle floor following ETV), extent of CPC (incomplete or complete), intraoperative bleeding (none, mild, moderate, severe), or neurological injury (e.g., injury to the fornix). To quantify and correlate the extent of CPC with outcome, the extent of subtotal CPC was subdivided into incomplete when CPC was unilateral or bilateral but only extended from the foramen of Monro (FM) to the atrium on one side. While we recognize that the complete choroid plexus (e.g., within the fourth ventricle, roof of third ventricle) cannot be obtained by CPC regardless of the endoscope used, we classified extent of CPC as being complete when CPC extended from the FM to the posterior temporal horn bilaterally (Table 1). Intraoperative bleeding was classified as follows: none, when there was no report of bleeding in the operation report; mild, when the surgeon reported minimal bleeding that was readily controlled with irrigation; moderate, when persistent bleeding requiring additional maneuvers to stop bleeding; and severe, when persistent severe bleeding requiring abortion of the procedure. The prepontine cistern was classified as scarred when the surgeon described abnormal, excessive scarring or arachnoid adhesions in the prepontine cistern following ETV/membrane of Liliequist fenestration, or as normal when the surgeon reported good visualization of the prepontine cistern and/or of the basilar artery after ETV/membrane of Liliequist fenestration.

The ETVSS ${ }^{24,28,32}$ and CCHU ETVSS ${ }^{53}$ were calculated for each patient using the appropriate variables (age, etiol- 


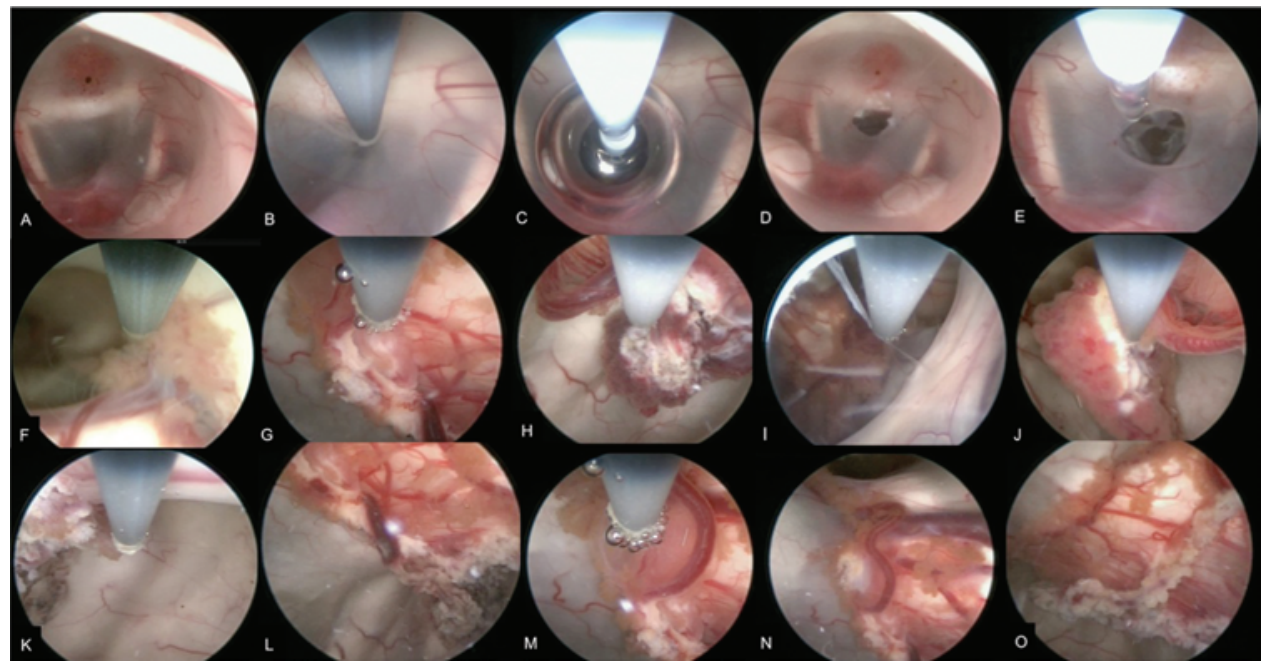

FIG. 1. Steps involved in ETV/CPC. Right frontal approach. Standard ETV with perforation of tuber cinereum (A-D) and membrane of Liliequist $(E)$. Cauterization of the right choroid plexus from the foramen of Monro $(F)$ toward the ipsilateral atrium ( $G$ and $\mathrm{H})$. Dehiscent septum pellucidum allowing visualization of the CP in the contralateral ventricle (I), which is progressively devascularized $(\mathrm{J})$ from the posterior temporal horn $(\mathrm{K})$ to the atrium $(\mathrm{L})$ and foramen of Monro (M and $\mathrm{N}$ ), until the CP is completely devascularized (0).

ogy, history of prior VPS, extent of CPC). For the CCHU ETVSS, based on ETV/CPC performed using a flexible endoscope, the extent of CPC is defined as none (0 points), partial unilateral ( 2 points), and complete bilateral (foramen of Monro to tip of temporal horns bilaterally, 4 points). Because a rigid neuroendoscope was used in our study, limiting the CPC from the foramen of Monro to the atrium/ posterior temporal horn, patients in whom complete CPC was obtained in our study were given a maximum score of 2 during calculation of the CCHU ETVSS, as there is residual choroid plexus in the temporal horns bilaterally.

\section{Outcome Measures}

Failure of ETV/CPC, the primary outcome measure, was defined as a repeated CSF diversion procedure (repeated ETV, reservoir requiring tapping, VPS) or death. Failure of ETV/CPC and the decision to perform a subsequent CSF diversion procedure (repeated ETV, CSF shunt, tapping of CSF reservoir) was made on an individual basis at each neurosurgeon's discretion. In general, this was based on typical clinical presentation of symptomatic raised intracranial pressure and/or neurological or neurodevelopmental stagnation or decline, in association with persistent or worsening ventriculomegaly. However, the symptomatology and presentation were not documented in this retrospective review. Procedure-related complications were also documented.

In patients with failed ETV/CPC undergoing CSF shunting, shunt survival was assessed using the time to event (TTE) method, in which an event was defined as shunt revision (e.g., shunt malfunction, infection) or death. The TTE was measured and calculated using the KaplanMeier survival method.

\section{Statistical Analysis}

We present the raw data using descriptive statistics. For continuous variables, we present medians, SDs, and range. For categorical data, we present frequencies and percentages. We created a Kaplan-Meier curve to demonstrate the overall time to ETV/CPC failure. We logarithmically transformed nonnormally distributed quantitative variables. We removed any variable with fewer than 20 observations. We performed univariate Cox regression analysis to determine if ETVSS and CCHU ETVSS were predic-

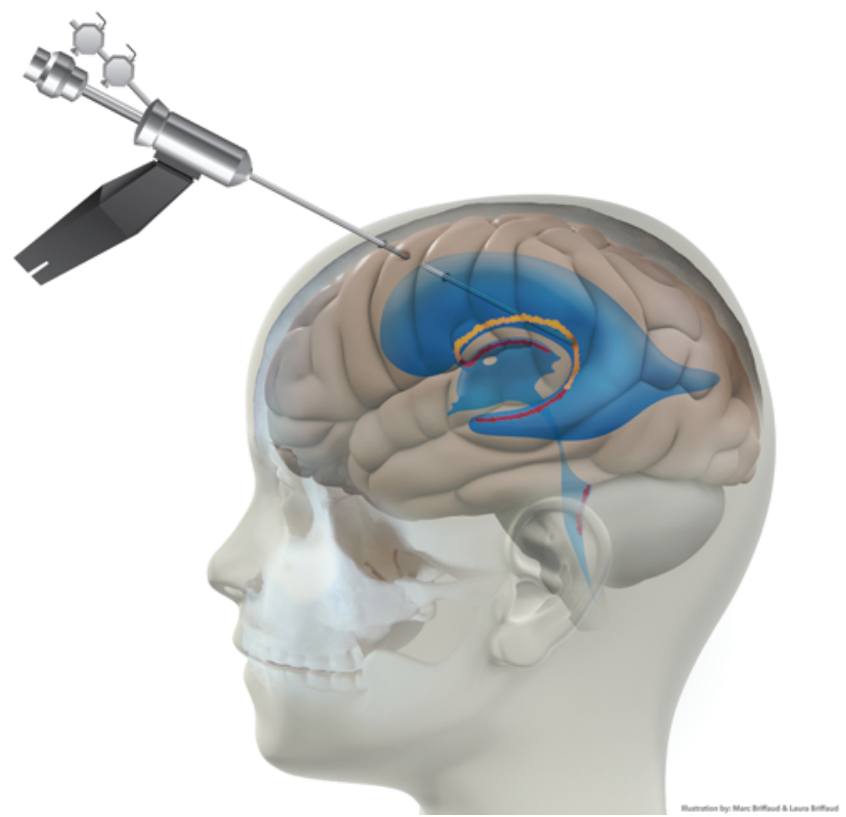

FIG. 2. Illustration demonstrating the typical maximal extent of CPC obtained with a rigid neuroendoscope, extending from the foramen of Monro to the posterior temporal horn (yellow choroid plexus). There is residual choroid plexus in the temporal horn in addition to the roof of the third and fourth ventricles (red choroid plexus). Copyright Alexander G. Weil. Published with permission. 


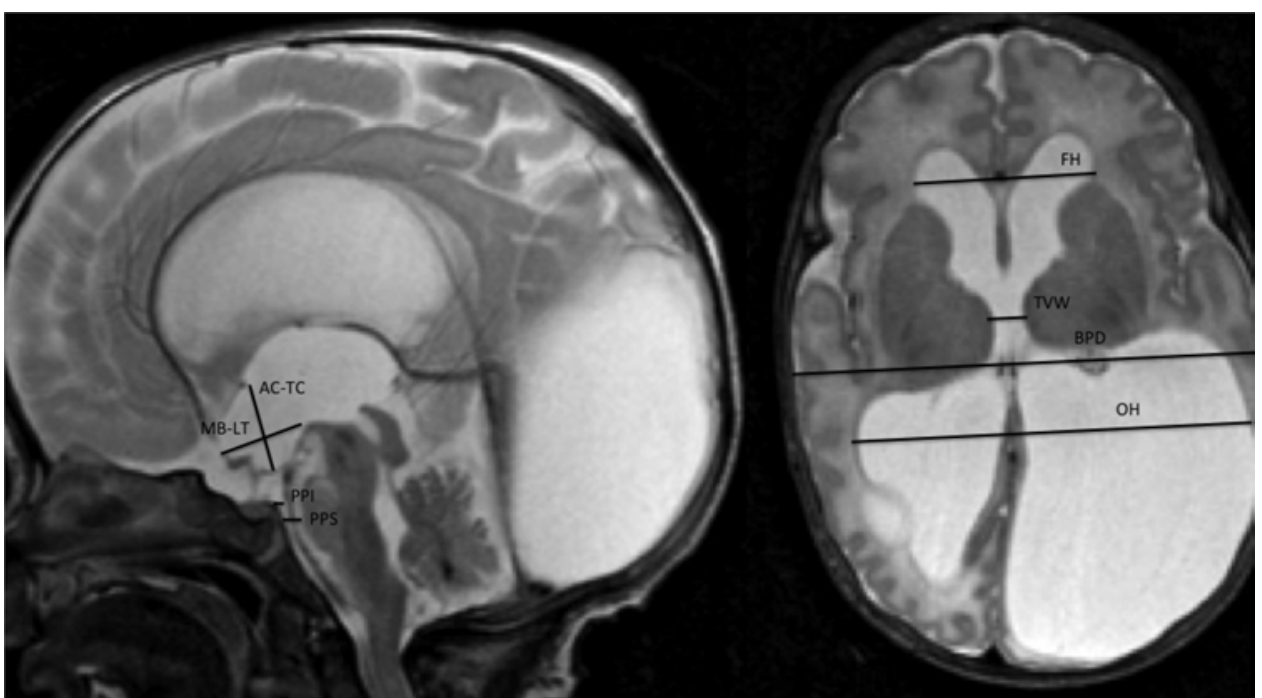

FIG. 3. Radiological measurements (in millimeters). Prepontine interval (PPI) from the dorsum sellae to the tip of the basilar artery. Prepontine space 6 from the midpons to clivus. Third Ventricular Morphology Index (TVMI), as calculated by adding the anterior commissure (AC) to tuber cinereum ${ }^{3}$ line (AC-TC) to the mammillary body to lamina terminalis line (MB-LT), and dividing by the biparietal diameter (BPD) [(AC-TC+MB-LT)/BPD]. The FOHR is calculated by adding the frontal horn (FH) and occipital horn $(\mathrm{OH})$ and divided by 2BPD [(FH+OH)/2BPD]. TVW = width of the third ventricle.

tive of ETV/CPC survival. We performed multivariate Cox regression analysis, adjusting for CCHU ETVSS, to determine the predictive suitability of multiple preoperative MR imaging (FOHR, TVW, TVMI, PPI, PPS, AS) and intraoperative (prepontine scarring, third ventriculostomy pulsations, extent of CPC) variables for ETV/CPC outcome. We created Kaplan-Meier curves to graph the overall time to ETV/CPC failure function. We accepted any variable with a $p$ value of less than 0.05 as a potential predictor of outcome. All statistics were performed in IBM SPSS Statistics version 20 (IBM Corp.).

\section{Results}

\section{Patient Population}

Between July 2007 and February 2014, 86 consecutive infants underwent ETV/CPC at Miami Children's Hospital/Jackson Memorial Hospital. Of these, 1 patient who was lost to follow-up was excluded from the study

\section{TABLE 1. Extent of CPC definitions}

\begin{tabular}{|c|c|c|c|}
\hline $\begin{array}{l}\text { Extent of } \\
\text { CPC }\end{array}$ & $\begin{array}{c}\text { Incomplete } \\
\text { (current study) }\end{array}$ & $\begin{array}{c}\text { Complete } \\
\text { (current study) }\end{array}$ & $\begin{array}{c}\text { Complete } \\
\text { (Warf et al., 2010) }\end{array}$ \\
\hline $\begin{array}{l}\text { Intraoperative } \\
\text { description } \\
\text { of CPC }\end{array}$ & $\begin{array}{l}\text { Unilateral CPC or } \\
\text { bilateral with } 1 \\
\text { side complete } \\
\text { and } 1 \text { extending } \\
\text { to FM or atrium }\end{array}$ & $\begin{array}{l}\text { Bilateral CPC } \\
\text { from FM to } \\
\text { posterior } \\
\text { TH }\end{array}$ & $\begin{array}{l}\text { Bilateral CPC } \\
\text { from FM to tip } \\
\text { of } \mathrm{TH}\end{array}$ \\
\hline $\begin{array}{l}\text { CCHU ET- } \\
\text { VSS extent } \\
\text { CPC grade }\end{array}$ & 2 & 2 & 4 \\
\hline
\end{tabular}

$\mathrm{FM}=$ foramen of Monro; $\mathrm{TH}=$ temporal horn. group. A total of 1, 2, 17, 26, 13, 11, 12, and 3 ETV/CPC procedures were performed in 2007, 2008, 2009, 2010, 2011, 2012, 2013, and 2014 (as of this writing), respectively. There were 45 boys and 40 girls with a mean age of 4.3 months (range 1 day to 20 months) (Table 2). Etiology included IVH of prematurity in 44 patients (51.7\%), MMC in 7 (8.2\%), congenital AS in $12(14.1 \%)$, congenital communicating hydrocephalus in $6(7.1 \%)$, Dandy-Walker complex in $6(7.1 \%)$, postinfectious in $5(5.8 \%)$, and other cause in $5(5.8 \%)$. Twenty-nine patients $(34.1 \%)$ had a previously placed reservoir (all IVH prematurity cases), while $5(5.8 \%)$ had a prior VPS and $8(9.4 \%)$ had a prior infection.

Seventy-six patients $(89.4 \%)$ had preoperative MRI data. The median (SD) FOHR was $0.57(0.07)$. The median $^{39}$ TVMI was 0.26 (11.90). The median ${ }^{39}$ PPI and PPS were $1.67 \mathrm{~mm}(1.90 \mathrm{~mm})$ and $3.54 \mathrm{~mm}(2.70 \mathrm{~mm})$, respectively. AS could be assessed in 75 of the 76 patients with imaging, and AS was present in 43 patients (57.3\%). The median $^{39}$ ETVSS and CCHU ETVSS were 40 (12.76) and 2 (0.96), respectively.

ETV and CPC were performed in all patients; extent of CPC was reported in 84 patients and was complete in 58 patients $(69.0 \%)$ and incomplete in $26(31.0 \%)$ (Table 3 ). Because extent of CPC was reported in a subtotal of patients but performed in all patients, the extent of CPC component of the CCHU ETVSS was 2 for all patients. Prepontine cistern status was reported in 83 patients and was described as scarred in $25(30.1 \%)$. Third ventricle pulsations were absent in 7 (13.2\%), present but moderate in 17 (32.0\%), and excellent in $29(54.7 \%)$ of the 53 cases in which it was reported. Intraoperative hemorrhage was absent in 70 patients $(82.3 \%)$, minimal in $13(15.3 \%)$, and moderate in $2(2.4 \%)$. There were no reports of inadvertent neurological injury. 
TABLE 2. Preoperative characteristics of 85 patients undergoing ETV/CPC

\begin{tabular}{|c|c|}
\hline Characteristic & Data* $^{*}$ \\
\hline \multicolumn{2}{|l|}{ Age at surgery in mos } \\
\hline$<1$ & $16(18.8)$ \\
\hline $1-6$ & $49(57.6)$ \\
\hline $6-12$ & $17(20)$ \\
\hline $12-24$ & $3(3.5)$ \\
\hline \multicolumn{2}{|l|}{ Etiology } \\
\hline IVH of prematurity & $44(51.8)$ \\
\hline MMC & $7(8.2)$ \\
\hline Congenital AS & $12(14.1)$ \\
\hline Congenital communicating hydrocephalus & $6(7)$ \\
\hline DWC & $6(7)$ \\
\hline Postinfectious & $5(5.9)$ \\
\hline Other etiology & $5(5.9)$ \\
\hline \multicolumn{2}{|l|}{ Prior CSF hardware } \\
\hline Prior reservoir & $29(34.1)$ \\
\hline Prior shunt & $5(5.8)$ \\
\hline Prior infection & $8(9.4)$ \\
\hline Preop MR images available & $76(89.4)$ \\
\hline \multicolumn{2}{|l|}{ Preop MR image score, median (SD) } \\
\hline FOHR & $0.57(0.07)$ \\
\hline TVMI & $0.26(11.9)$ \\
\hline PPI & $1.67(1.9)$ \\
\hline PPS & $3.54(2.7)$ \\
\hline Presence of AS $(n=75)$ & $43(57.3)$ \\
\hline \multicolumn{2}{|l|}{ Scoring system, median score (SD) } \\
\hline ETVSS & $40(12.76)$ \\
\hline CCHU ETVSS & $2(0.96)$ \\
\hline
\end{tabular}

DWC $=$ Dandy-Walker complex .

${ }^{*}$ Data given as number of patients (\%) unless otherwise indicated.

\section{ETV/CPC Outcome}

ETV/CPC was successful in $42.1 \%, 37.7 \%$, and $36.8 \%$ of patients at 6,12 , and 24 months of follow-up, respectively (Fig. 4). The median (95\% CI) time to ETV/CPC failure was 4.0 months (0.93-7.07 months). In univariate analyses, both the ETVSS (hazard ratio [HR] 1.03, 95\% CI $1.01-1.05, \mathrm{p}=0.004$ ) and CCHU ETVSS (HR 1.48; 95\% CI $1.04-2.09 ; \mathrm{p}=0.028$ ) were predictive of outcome (Table 4). In multivariate analysis, when adjusting for CCHU ETVSS, the presence of prepontine scarring was associated with ETV/CPC failure (HR 0.34; 95\% CI 0.19-0.63; $\mathrm{p}<0.001)$. Other variables, such as radiological criteria (PPI, PPS, AS, TVMI) and intraoperative findings (ventriculostomy pulsations, extent of $\mathrm{CPC}$ ), did not predict success (Table 5).

Postoperative MRI was available for review in 75 patients (88.2\%) at a mean follow-up of 2.9 months (range 1-72 months). The median FOHR ${ }^{39}$ was 0.49 (0.11). The median TVMI ${ }^{39}$ was $0.18(0.05)$. In patients with successful ETV/CPC, the median ${ }^{39}$ FOHR was $0.52(0.09)$ compared with $0.45(0.12)$ in patients who failed ETV/CPC and
TABLE 3. Intraoperative variables in 85 patients undergoing ETVI CPC

\begin{tabular}{lc}
\hline \multicolumn{1}{c}{ Variable } & No. $(\%)$ \\
\hline ETV & $85(100)$ \\
\hline Performed & $25(30.1)$ \\
\hline Prepontine cistern status $(\mathrm{n}=83)$ & \\
\hline Scarring present & $29(54.7)$ \\
\hline Ventilation of third ventricle $(\mathrm{n}=53)$ & $17(32)$ \\
\hline Excellent & $7(13.2)$ \\
\hline Mild & $77(90.6)$ \\
\hline None & $26(31)$ \\
\hline Septostomy $(\mathrm{n}=84)$ & $58(69)$ \\
\hline Performed or already present & \\
\hline Extent of CPC $(\mathrm{n}=84)$ & $70(82.3)$ \\
\hline Incomplete & $13(15.3)$ \\
\hline Complete & $2(2.4)$ \\
\hline Intraoperative bleeding $(\mathrm{n}=85)$ & $0(0)$ \\
\hline None &
\end{tabular}

were subsequently treated by placement of CSF shunts ( $\mathrm{p}$ $<0.005$ ). In patients in whom ETV/CPC was successful, the median ${ }^{39}$ TVMI was $0.20(0.05)$ compared with 0.16 $(0.05)$ in patients subsequently treated with placement of CSF shunts for ETV/CPC failure $(\mathrm{p}<0.001)$.

There were 6 procedure-related complications in 5 patients (5.8\%), all occurring in the first half of the study period (before May 2010) (Table 6). Two patients developed subdural hygromas, one of which was symptomatic requiring aspiration. Three patients developed meningitis, 1 in the context of a CSF leak. All of these patients had a history of IVH of prematurity with CSF reservoir prior to ETV/CPC. Two of the infections occurred within 2 weeks following ETV/CPC and both required subsequent VPS placement. In the third case, meningitis occurred after VPS placed 2 weeks after ETV/CPC. The VPS infection was treated with VPS removal, CSF reservoir placement, and serial CSF aspirations; however, the patient developed a cerebral abscess requiring surgical evacuation. This patient died 2 months postoperatively. Overall, there were 3 deaths (3.5\%) in this series; these occurred at 2, 8 , and 26 months after surgery. Two of the mortalities occurred 6 weeks and 21 months after a VPS was placed for ETV/ CPC failure. The other mortality was a newborn with hydranencephaly who died of cardiorespiratory arrest 8 months after ETV/CPC.

\section{Shunt Survival After Failed ETV/CPC}

Of the 49 patients with failed ETV/CPC, 48 underwent VPS; of these, 46 had follow-up after shunting. Overall, CSF shunting was successful in $95.6 \%, 95.6 \%$, and $87.0 \%$ of patients at 6,12 , and 24 months follow-up, respectively (Fig. 5). The median (95\% CI) time to ETV/CPC failure 


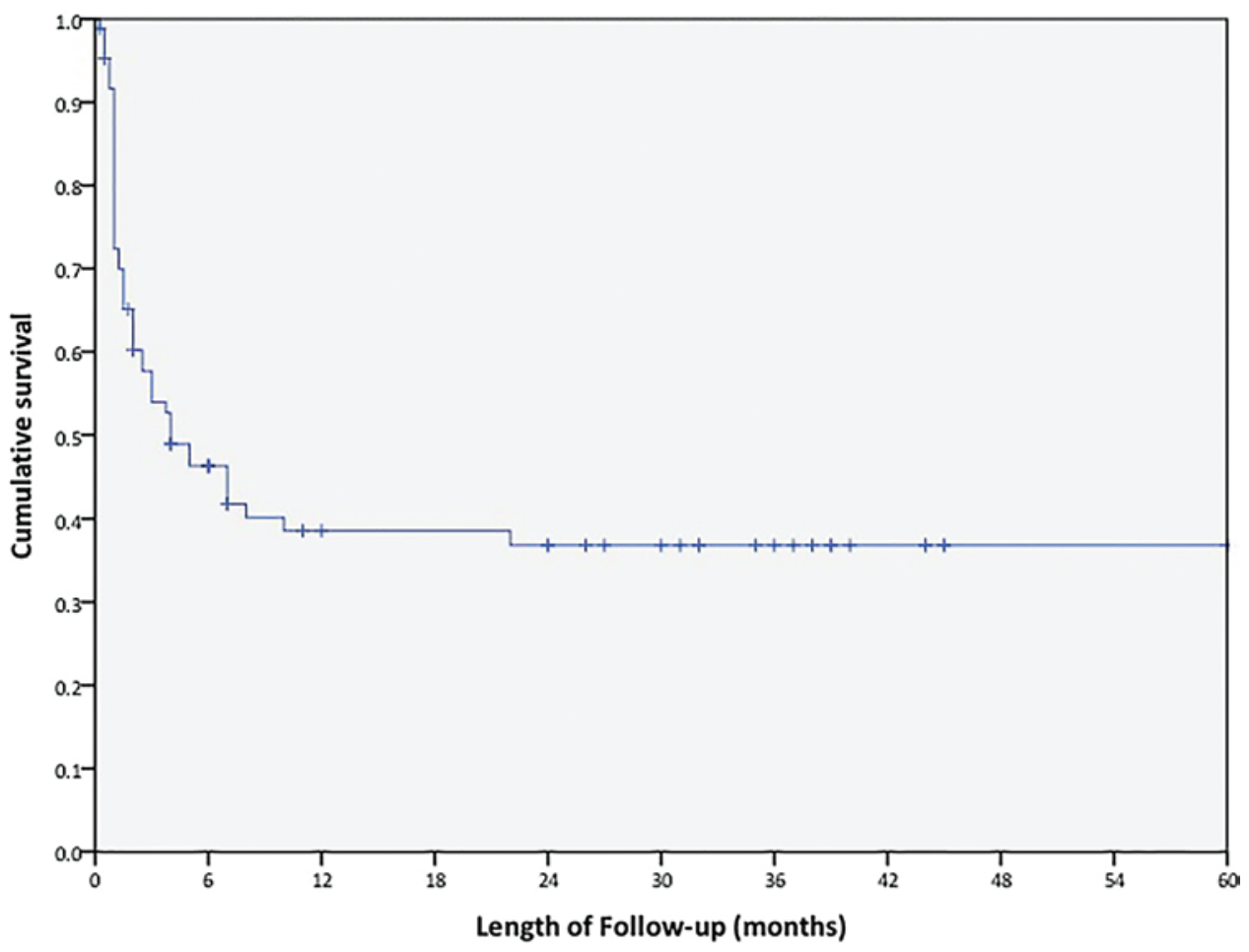

FIG. 4. Kaplan-Meier survival curve of time to ETVICPC failure. Figure is available in color online only.

was 60.4 months (52.8-68.0 months). The "events" included 1 death; shunt infections in 2 patients (4.4\%), 1 of which occurred from a perforated appendicitis; and shunt malfunctions, due to obstruction, in 3 patients $(6.6 \%$; 5 revisions).

\section{Discussion}

We report a single-center North American experience with ETV/CPC for hydrocephalus of infancy. Our first objective was to evaluate the safety and efficacy of this procedure for North American infants with hydrocephalus. Our secondary objective was to assess whether the ETVSS, the CCHU ETVSS, or other factors could predict success, to identify ideal candidates for ETV/CPC.

\section{Is ETV/CPC Safe?}

For it to be considered as a first-line treatment for infantile hydrocephalus, the complication profile of ETV/ CPC must be equivalent to or better than that of ETV or CSF shunting. The complication profile in this study compares favorably to prior series of ETV/CPC in both North American ${ }^{5,29,50}$ and developing countries. ${ }^{46}$ (Table 7). Our study corroborates others in suggesting the addi-

TABLE 4. Univariate analysis of scoring system variables for ETV/CPC success

\begin{tabular}{lccc}
\hline Scoring System & HR & $95 \% \mathrm{Cl}$ & $\mathrm{p}$ Value \\
\hline ETVSS & 1.013 & $1.010-1.054$ & 0.004 \\
\hline CCHU ETVSS & 1.477 & $1.043-2.092$ & 0.028 \\
\hline
\end{tabular}

tion of CPC does not lead to an increase in complications compared with ETV performed alone in infants, where the reported rate of meningitis is $0 \%-12.0 \%$, including CSF leak in $0 \%-8.5 \%$, hemorrhage in $0 \%-4.3 \%$, and hygroma in $0 \%-4.7 \%$. $110,11,13,14,17,20,34,45$ However, the mortality rate of $3.5 \%$ in our series is not negligible, and, although similar to that reported by Kulkarni et al. (8.3\%), ${ }^{29}$ it is higher than mortality reported in series of infants undergoing ETV/ CPC in other North American centers $(0 \%),{ }^{44}$ in Uganda $(1.0 \%)$, and following ETV alone worldwide, where it is closer to $0 \%$. $, 2,10,11,13,14,20,34,45$ However, comparison between cohorts of patients undergoing ETV/CPC, ETV, or CSF shunting from different studies and centers is severely lim-

TABLE 5. Multivariate analysis of variables for ETVICPC success adjusted for CCHU ETVSS

\begin{tabular}{lccc}
\hline \multicolumn{1}{c}{ Characteristic } & $\mathrm{HR}$ & $95 \% \mathrm{Cl}$ & $\mathrm{p} \mathrm{Value}$ \\
\hline Preoperative & & & \\
\hline PPI & 1.001 & $0.850-1.179$ & 0.987 \\
\hline PPS & 0.941 & $0.828-1.069$ & 0.348 \\
\hline TVW & 1.034 & $0.979-1.092$ & 0.227 \\
\hline TVMI & 1.015 & $0.996-1.036$ & 0.130 \\
\hline FOHR & 0.067 & $0.001-7.842$ & 0.067 \\
\hline Intraoperative & & & \\
\hline Extent of CPC & 1.030 & $0.550-1.928$ & 0.928 \\
\hline Prepontine scarring & 0.344 & $0.189-0.625$ & $<0.001^{*}$ \\
\hline Third ventricular floor pulsation & 1.451 & $0.651-3.235$ & 0.363 \\
\hline *Statistically significant. & & &
\end{tabular}


TABLE 6. Postoperative variables in 85 patients undergoing ETV/CPC

\begin{tabular}{lc}
\hline \multicolumn{1}{c}{ Characteristic } & Data \\
\hline Complications, $\mathrm{n}(\%)$ & \\
\hline Infection & $3(3.5)$ \\
\hline Hygroma & $2(2.3)$ \\
\hline CSF leak & $1(1.2)$ \\
\hline Postoperative MRI available, $\mathrm{n}(\%)$ & $75(88.2)$ \\
\hline Mean follow-up in mos (range) & $2.9(1-72)$ \\
\hline Median FOHR (SD) & $0.49(0.11)$ \\
\hline Median TVMI (SD) & $0.18(0.05)$ \\
\hline
\end{tabular}

ited by differences in the patient populations. Differences in mortality are likely heavily influenced by this selection bias, as patients undergoing ETV/CPC suffer from more complex underlying conditions (e.g., IVH of prematurity, hydranencephaly) than those undergoing standard ETV. The mortality is related to these underlying conditions, as none of the mortalities in our series were directly related to the ETV/CPC procedure itself: 2 occurred after VPS placement and 1 was related to the underlying condition (e.g., hydranencephaly) of the child.

\section{What Is the Role of ETV/CPC?}

The success rate of ETV/CPC in this single-center cohort is similar to that of many other North American studies. ${ }^{5,29,50}$ The 12-month success rate of $37 \%$ is lower than that recently reported by Kulkarni et al. (52\%) and Stone and Warf (57\%), both of which were North American studies. ${ }^{29,44}$ However, comparison between studies is limited by differences in patient population and nonstandardized methodology and criteria for undergoing a repeated CSF diversion procedure. ${ }^{29,44}$ For example, compared with Kulkarni et al., our series had a higher proportion of patients younger than 6 months ( $76 \%$ vs 56\%), more patients with prior IVH of prematurity (52\% vs $25 \%$ ), and a lower predicted success rate based on the median CCHU ETVSS (2 vs 4) and ETVSS (40 vs 50). ${ }^{29}$ Also, compared with Stone and Warf, our series had a higher proportion of patients with unfavorable risk factors, such as age less than 6 months (76.4\% vs $68.0 \%)$, prepontine scarring (30.1\% vs $16.5 \%)$, and more patients with IVH of prematurity $(51.8 \%$ vs $27.5 \%$ ), limiting direct comparison of outcome data.

This study found that ETV/CPC has a failure profile similar to ETV, with a higher rate of early postoperative failure compared with shunting. ${ }^{16,21,25}$ The 6- and 12-month ETV/CPC success rates of $41 \%$ and $38 \%$, respectively, in our series are inferior to the VPS survival rates of $62 \%$ and $47 \%$, respectively. ${ }^{19,25}$ However, while the ETV/CPC success rate stabilizes at $37 \%$ after 2 years, shunt survival rates drop to $41 \%$ by 4 years and $20 \%$ by 12 years, suggesting that any difference in efficacy between ETV/CPC and shunting may become very small in the long term..$^{19,29}$

Some authors have questioned the relative contribution of CPC in improving success rates for ETV in infants. ${ }^{41}$ The overall success rate of ETV/CPC performed across several North American centers has not systematically been superior to that reported for ETV.,10,11,13,14,16,17,20,34,45 However, comparisons of results of ETV/CPC from our single center to historical controls of ETV alone for infantile hydrocephalus, including more recent studies, are significantly

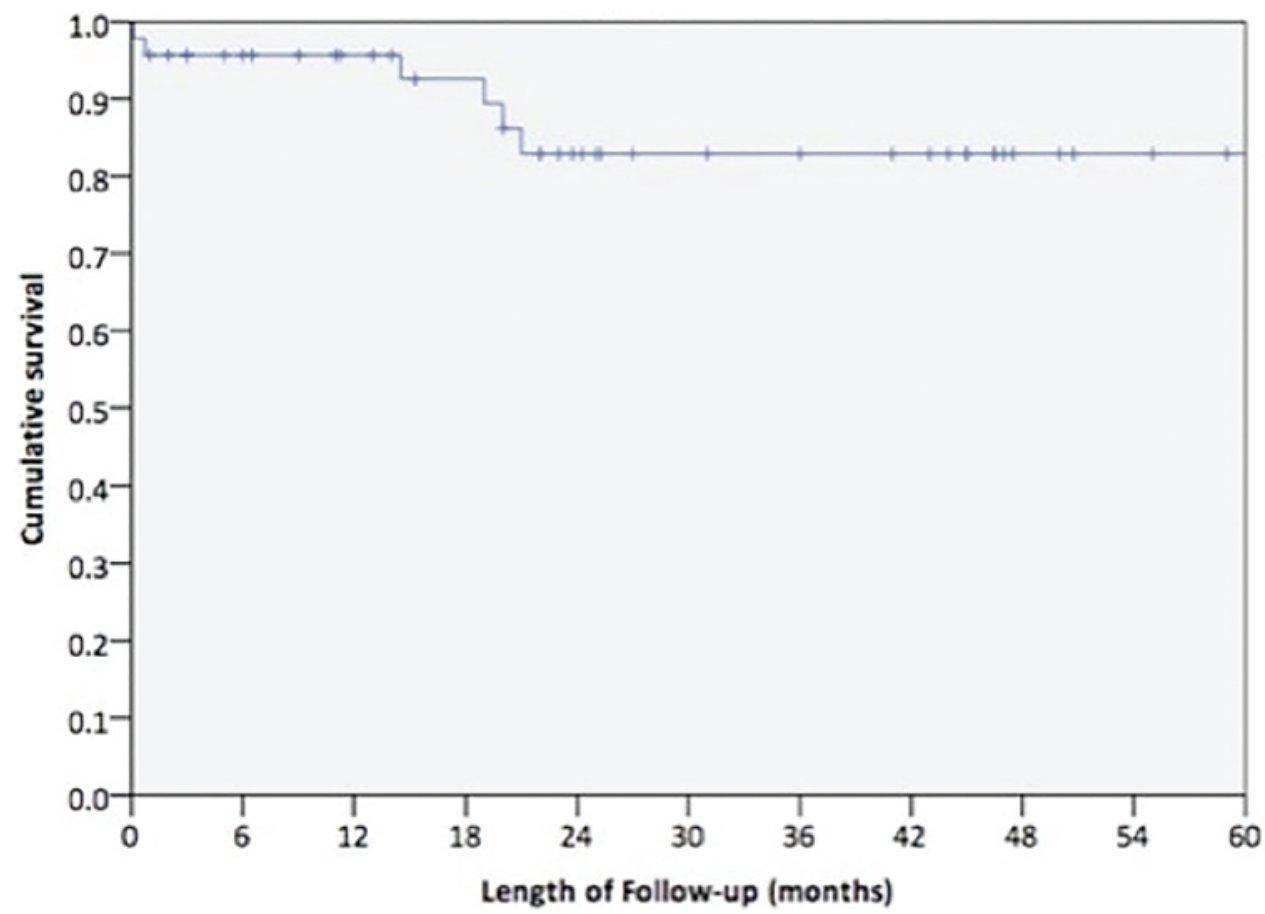

FIG. 5. Time to shunt failure. Kaplan-Meier survival curve of CSF shunt survival after failed ETVICPC. Figure is available in color online only. 


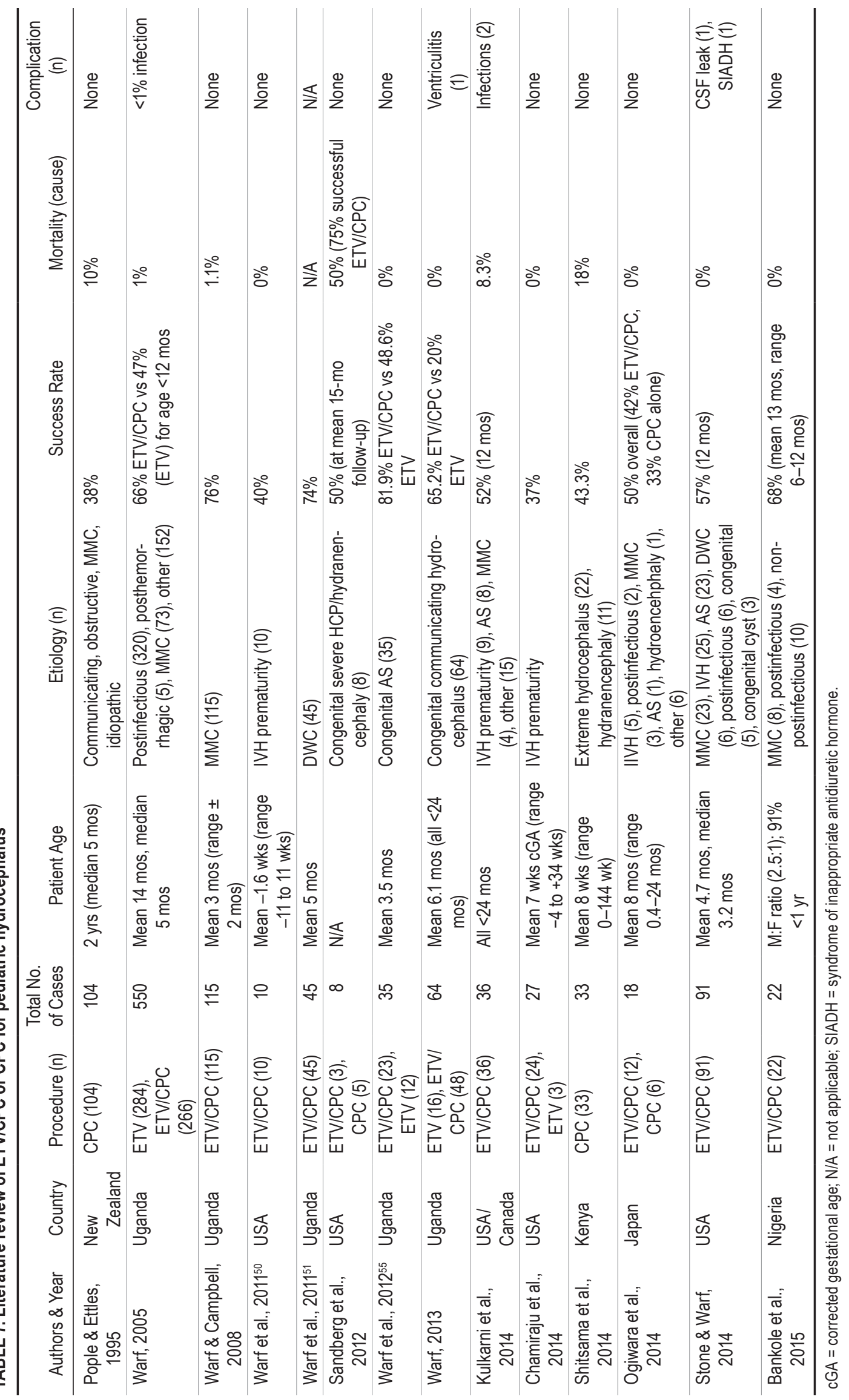


limited by many uncontrolled variables. ${ }^{16}$ In many studies, the success rate of ETV/CPC has also not been shown to be superior to the success rate predicted by the ETVSS, which predicts success following ETV alone. ${ }^{5,29,50}$ For example, the mean ETVSS was $40 \%$ in our series, which is roughly equivalent to the actual predicted 6-month ETV/ CPC success of $42.1 \%$. This would suggest a modest benefit at best. However, a more recent North American study of 91 infants undergoing ETV/CPC found that the 6-month success rate of $59 \%$ exceeded the $45 \%$ rate predicted by the ETVSS. ${ }^{44}$ Furthermore, some authors have shown standalone CPC (without ETV) to be a relatively efficient treatment with good (38\%) long-term efficacy in children with hydrocephalus. ${ }^{36}$ Finally, a study by Warf in sub-Saharan Africa strongly suggests an important role for CPC, as it has been found to almost double the efficacy of ETV in patients with MMC, AS, and DWC, and to triple efficacy in cases of postinfectious or communicating hydrocephalus. ${ }^{47,49,51,55}$ The finding of a dose response of CPC, in which more extensive CPC improves success rate over less extensive CPC, as reported by Warf, also supports the role of $\mathrm{CPC}$ and is the basis for the CCHU ETVSS. ${ }^{53}$

\section{Selecting a Candidate for ETV/CPC}

This is the first study to show that the ETVSS can predict success following ETV/CPC. It is also the first study to show that the CCHU ETVSS can predict success after ETV/CPC in a North American cohort of infants. The ETVSS was specifically designed and validated to predict success in patients undergoing ETV. ${ }^{26}$ The CCHU ETVSS was developed based on data from ETV/CPC performed using a flexible endoscope on children at the CCHU in sub-Saharan Africa. ${ }^{53}$ This scale includes an assessment of the extent of CPC, in addition to age and etiology; however, since all our patients had an extent of CPC score 2 (incomplete), only etiology and age were contributing factors. Overall, this suggests that age and etiology are likely important determinants of success. A recent study has shown postinfectious hydrocephalus to be associated with ETV/CPC failure. ${ }^{44}$ Although these findings suggest that both these scoring systems can predict which patients will fail and help patient selection in patients treated at our center, whether these results are generalizable to other centers remains unknown. ${ }^{29}$ A recent multi-institutional study of 36 infants undergoing ETV/CPC failed to demonstrate any impact of age or etiology. ${ }^{29}$ Both the ETVSS and CCHU ETVSS also failed to predict success, suggesting that further studies are warranted to verify if our results are generalizable. ${ }^{29}$

Not surprisingly, prepontine scarring predicted ETV/ $\mathrm{CPC}$ failure, as has been shown by other authors when assessed on preoperative $\mathrm{MRI}^{50}$ and during surgery. ${ }^{44,52}$ This suggests that in the presence of prepontine scarring, the CPC may not decrease CSF production sufficiently to treat the hydrocephalus, underscoring the important role the concomitant ETV plays in ETV/CPC.

\section{Does Extent of CPC Matter?}

How much of the choroid plexus must be cauterized for a successful ETV/CPC is unknown. The extent of CPC necessary to improve success rate is also controversial. ${ }^{5,44}$
Some authors consider subtotal CPC with a rigid neuroendoscope to be sufficient, ${ }^{5}$ while others advocate performing complete CPC with a flexible neuroendoscope to optimize efficacy. ${ }^{44,49}$ In support of Warf's findings, Kulkarni et al. recently found a trend $(\mathrm{p}=0.0501)$ for improved success rate, above that predicted by the ETVSS, following near-complete CPC (greater than 90\%) in a mixed etiology cohort of 36 North American infants undergoing ETV/ CPC. ${ }^{29}$ A more recent North American study in 91 infants showed a higher 57\% 12-month success rate in infants undergoing ETV/CPC, using a flexible neuroendoscope, suggesting a possible added benefit of complete CPC. ${ }^{44}$ However, the extent of CPC was assessed subjectively by the operating neurosurgeon, which is known to be an unreliable assessment in the tumor literature, with frequent overestimation of extent of resection. ${ }^{56}$ None of these studies on ETV/CPC has evaluated the extent of CPC in an objective, independent, or blinded fashion using objective biomarkers (e.g., MRI to evaluate the choroid plexus postoperatively). Finally, the literature on ETV/CPC for infantile hydrocephalus related to IVH of prematurity shows a $40 \%$ success rate regardless of whether flexible endoscope-guided complete CPC or rigid endoscope-guided subtotal CPC is performed. ${ }^{5,50}$ While the ultimate goal of ETV/CPC is to alter the balance of CSF production and absorption sufficient enough to avoid a CSF diversion, the optimal extent of CPC may be different for each patient, with some patients requiring a more extensive CPC than others.

\section{Defining Success}

Although our primary end point of shunt independence is the most commonly used end point to assess efficacy of ETV or ETV/CPC, ${ }^{24}$ it does not provide information on the patient's functional outcome. Although unavailable in this retrospective chart review, neurocognitive outcome and health-related quality of life may have been more informative and relevant end points..$^{27,41}$ Is it better to live with shunt-independent ventriculomegaly following a successful ETV/CPC than to survive with small- to normal-sized shunted ventricles, with the well-established long-term risk of shunt infection, malfunction, and reoperation? ${ }^{7,43}$ Avoiding multiple shunt infections and surgical revisions should be a primary objective in infants with hydrocephalus, as they have been shown to correlate with a worse neurocognitive outcome. ${ }^{31,37}$ Furthermore, several authors have shown that in the absence of raised intracranial pressure, ventriculomegaly does not impair neurodevelopment. ${ }^{15,54}$ This is supported by Warf et al., who demonstrated comparable neurocognitive outcomes in hydrocephalic MMC children undergoing ETV/CPC or CSF shunting. ${ }^{54}$

Some authors have questioned whether the CPC, which alters CSF composition and physiology in unknown ways, can predispose patients who fail and go on to have shunts placed to a higher rate of complications. ${ }^{41}$ The rate of shunt failure in the patients shunted after failed ETV/CPC was comparable to other series looking at shunted patients following failed $\mathrm{CPC}^{36}$ and within the range expected following primary shunt placement. ${ }^{7,19}$ Although these data are anecdotal, they are supported by a recent study in sub-Saharan hydrocephalic infants that showed that the addition 
of CPC to ETV did not affect shunt survival and infection rate compared with patients in whom a shunt was placed primarily or after ETV alone. ${ }^{48}$

\section{Study Limitations}

There are multiple limitations to this study that are inherent to its retrospective nature, small sample size, and heterogeneous patient population. The nonblinded data collection introduces a bias. Although this is a consecutive series of patients undergoing ETV/CPC, other patients underwent either ETV or CSF shunting at our institution during the study period, introducing a selection bias. Because of this, and because it is a single-center experience, whether the results are generalizable to other centers with different surgeons, techniques, and patient populations with hydrocephalus is unknown.

\section{Conclusions}

ETV/CPC is a feasible alternative to ETV and VPS in infants with hydrocephalus. Both the ETVSS and CCHU ETVSS predicted success following ETV/CPC in this single-center North American cohort. The presence of prepontine scarring may reduce success after this procedure.

\section{References}

1. Baldauf J, Oertel J, Gaab MR, Schroeder HW: Endoscopic third ventriculostomy in children younger than 2 years of age. Childs Nerv Syst 23:623-626, 2007

2. Bankole OB, Ojo OA, Nnadi MN, Kanu OO, Olatosi JO: Early outcome of combined endoscopic third ventriculostomy and choroid plexus cauterization in childhood hydrocephalus. J Neurosurg Pediatr 15:524-528, 2015

3. Bracken MB, Shepard MJ, Holford TR, Leo-Summers L, Aldrich EF, Fazl M, et al: Administration of methylprednisolone for 24 or 48 hours or tirilazad mesylate for 48 hours in the treatment of acute spinal cord injury. Results of the Third National Acute Spinal Cord Injury Randomized Controlled Trial. National Acute Spinal Cord Injury Study. JAMA 277:1597-1604, 1997

4. Buxton N, Macarthur D, Mallucci C, Punt J, Vloeberghs M: Neuroendoscopic third ventriculostomy in patients less than 1 year old. Pediatr Neurosurg 29:73-76, 1998

5. Chamiraju P, Bhatia S, Sandberg DI, Ragheb J: Endoscopic third ventriculostomy and choroid plexus cauterization in posthemorrhagic hydrocephalus of prematurity. J Neurosurg Pediatr 13:433-439, 2014

6. Clark KL, Hughes SA, Bulsara P, Coates J, Moores K, Parry $\mathrm{J}$, et al: Pharmacological characterization of a novel ENaCa siRNA (GSK2225745) with potential for the treatment of cystic fibrosis. Mol Ther Nucleic Acids 2:e65, 2013

7. Drake JM, Kestle JR, Milner R, Cinalli G, Boop F, Piatt J Jr, et al: Randomized trial of cerebrospinal fluid shunt valve design in pediatric hydrocephalus. Neurosurgery 43:294-305, 1998

8. Drake JM, Kulkarni AV, Kestle J: Endoscopic third ventriculostomy versus ventriculoperitoneal shunt in pediatric patients: a decision analysis. Childs Nerv Syst 25:467-472, 2009

9. Durnford AJ, Kirkham FJ, Mathad N, Sparrow OC: Endoscopic third ventriculostomy in the treatment of childhood hydrocephalus: validation of a success score that predicts long-term outcome. J Neurosurg Pediatr 8:489-493, 2011

10. Faggin R, Bernardo A, Stieg P, Perilongo G, d'Avella D: Hydrocephalus in infants less than six months of age: effec- tiveness of endoscopic third ventriculostomy. Eur J Pediatr Surg 19:216-219, 2009

11. Fani L, de Jong TH, Dammers R, van Veelen ML: Endoscopic third ventriculocisternostomy in hydrocephalic children under 2 years of age: appropriate or not? A single-center retrospective cohort study. Childs Nerv Syst 29:419-423, 2013

12. Foroughi M, Wong A, Steinbok P, Singhal A, Sargent MA, Cochrane DD: Third ventricular shape: a predictor of endoscopic third ventriculostomy success in pediatric patients. J Neurosurg Pediatr 7:389-396, 2011

13. Fritsch MJ, Kienke S, Ankermann T, Padoin M, Mehdorn HM: Endoscopic third ventriculostomy in infants. J Neurosurg 103 (1 Suppl):50-53, 2005

14. Gallo P, Szathmari A, De Biasi S, Mottolese C: Endoscopic third ventriculostomy in obstructive infantile hydrocephalus: remarks about the so-called 'unsuccessful cases'. Pediatr Neurosurg 46:435-441, 2010

15. Hanlo PW, Gooskens RJ, van Schooneveld M, Tulleken CA, van der Knaap MS, Faber JA, et al: The effect of intracranial pressure on myelination and the relationship with neurodevelopment in infantile hydrocephalus. Dev Med Child Neurol 39:286-291, 1997

16. He Z, An C, Zhang X, He X, Li Q: The efficacy analysis of endoscopic third ventriculostomy in infantile hydrocephalus. J Korean Neurosurg Soc 57:119-122, 2015

17. Javadpour M, Mallucci C, Brodbelt A, Golash A, May P: The impact of endoscopic third ventriculostomy on the management of newly diagnosed hydrocephalus in infants. Pediatr Neurosurg 35:131-135, 2001

18. Kadrian D, van Gelder J, Florida D, Jones R, Vonau M, Teo $\mathrm{C}$, et al: Long-term reliability of endoscopic third ventriculostomy. Neurosurgery 62 (Suppl 2):614-621, 2008

19. Kestle J, Drake J, Milner R, Sainte-Rose C, Cinalli G, Boop F, et al: Long-term follow-up data from the Shunt Design Trial. Pediatr Neurosurg 33:230-236, 2000

20. Koch D, Wagner W: Endoscopic third ventriculostomy in infants of less than 1 year of age: which factors influence the outcome? Childs Nerv Syst 20:405-411, 2004

21. Koch-Wiewrodt D, Wagner W: Success and failure of endoscopic third ventriculostomy in young infants: are there different age distributions? Childs Nerv Syst 22:1537-1541, 2006

22. Kulkarni AV, Drake JM, Armstrong DC, Dirks PB: Imaging correlates of successful endoscopic third ventriculostomy. J Neurosurg 92:915-919, 2000

23. Kulkarni AV, Drake JM, Armstrong DC, Dirks PB: Measurement of ventricular size: reliability of the frontal and occipital horn ratio compared to subjective assessment. Pediatr Neurosurg 31:65-70, 1999

24. Kulkarni AV, Drake JM, Kestle JR, Mallucci CL, Sgouros S, Constantini S: Predicting who will benefit from endoscopic third ventriculostomy compared with shunt insertion in childhood hydrocephalus using the ETV Success Score. J Neurosurg Pediatr 6:310-315, 2010

25. Kulkarni AV, Drake JM, Kestle JR, Mallucci CL, Sgouros S, Constantini S: Endoscopic third ventriculostomy vs cerebrospinal fluid shunt in the treatment of hydrocephalus in children: a propensity score-adjusted analysis. Neurosurgery 67:588-593, 2010

26. Kulkarni AV, Drake JM, Mallucci CL, Sgouros S, Roth J, Constantini S: Endoscopic third ventriculostomy in the treatment of childhood hydrocephalus. J Pediatr 155:254-9.e1, 2009

27. Kulkarni AV, Hui S, Shams I, Donnelly R: Quality of life in obstructive hydrocephalus: endoscopic third ventriculostomy compared to cerebrospinal fluid shunt. Childs Nerv Syst 26:75-79, 2010

28. Kulkarni AV, Riva-Cambrin J, Browd SR: Use of the ETV Success Score to explain the variation in reported endoscopic 
third ventriculostomy success rates among published case series of childhood hydrocephalus. J Neurosurg Pediatr 7:143-146, 2011

29. Kulkarni AV, Riva-Cambrin J, Browd SR, Drake JM, Holubkov R, Kestle JR, et al: Endoscopic third ventriculostomy and choroid plexus cauterization in infants with hydrocephalus: a retrospective Hydrocephalus Clinical Research Network study. J Neurosurg Pediatr 14:224-229, 2014

30. Kulkarni AV, Warf BC, Drake JM, Mallucci CL, Sgouros S, Constantini S: Surgery for hydrocephalus in sub-Saharan Africa versus developed nations: a risk-adjusted comparison of outcome. Childs Nerv Syst 26:1711-1717, 2010

31. Levy ML, Masri LS, McComb JG: Outcome for preterm infants with germinal matrix hemorrhage and progressive hydrocephalus. Neurosurgery 41:1111-1118, 1997

32. Naftel RP, Reed GT, Kulkarni AV, Wellons JC: Evaluating the Children's Hospital of Alabama endoscopic third ventriculostomy experience using the Endoscopic Third Ventriculostomy Success Score: an external validation study. J Neurosurg Pediatr 8:494-501, 2011

33. O'Hayon BB, Drake JM, Ossip MG, Tuli S, Clarke M: Frontal and occipital horn ratio: A linear estimate of ventricular size for multiple imaging modalities in pediatric hydrocephalus. Pediatr Neurosurg 29:245-249, 1998

34. Ogiwara H, Dipatri AJ Jr, Alden TD, Bowman RM, Tomita T: Endoscopic third ventriculostomy for obstructive hydrocephalus in children younger than 6 months of age. Childs Nerv Syst 26:343-347, 2010

35. Ogiwara H, Uematsu K, Morota N: Obliteration of the choroid plexus after endoscopic coagulation. J Neurosurg Pediatr 14:230-233, 2014

36. Pople IK, Ettles D: The role of endoscopic choroid plexus coagulation in the management of hydrocephalus. Neurosurgery 36:698-702, 1995

37. Resch B, Gedermann A, Maurer U, Ritschl E, Müller W: Neurodevelopmental outcome of hydrocephalus following intra-/periventricular hemorrhage in preterm infants: shortand long-term results. Childs Nerv Syst 12:27-33, 1996

38. Sandberg DI, Chamiraju P, Zoeller G, Bhatia S, Ragheb J: Endoscopic choroid plexus coagulation in infants with hydranencephaly or hydrocephalus with a minimal cortical mantle. Pediatr Neurosurg 48:6-12, 2012

39. Santosh C, Teasdale E, Molyneux A: Spontaneous closure of an intracranial middle cerebral arteriovenous fistula. Neuroradiology 33:65-66, 1991

40. Shitsama S, Wittayanakorn N, Okechi H, Albright AL: Choroid plexus coagulation in infants with extreme hydrocephalus or hydranencephaly. J Neurosurg Pediatr 14:55-57, 2014

41. Souweidane MS: Combined choroid plexus coagulation and endoscopic third ventriculostomy: is North America ready? J Neurosurg Pediatr 14:221-223, 2014

42. Souweidane MM, Morgenstern PF, Kang S, Tsiouris AJ, Roth $\mathrm{J}$ : Endoscopic third ventriculostomy in patients with a diminished prepontine interval. J Neurosurg Pediatr 5:250-254, 2010

43. St George E, Natarajan K, Sgouros S: Changes in ventricular volume in hydrocephalic children following successful endoscopic third ventriculostomy. Childs Nerv Syst 20:834-838, 2004

44. Stone SS, Warf BC: Combined endoscopic third ventriculostomy and choroid plexus cauterization as primary treatment for infant hydrocephalus: a prospective North American series. J Neurosurg Pediatr 14:439-446, 2014

45. Sufianov AA, Sufianova GZ, Iakimov IA: Endoscopic third ventriculostomy in patients younger than 2 years: outcome analysis of 41 hydrocephalus cases. J Neurosurg Pediatr 5:392-401, 2010

46. Warf BC: Comparison of endoscopic third ventriculostomy alone and combined with choroid plexus cauterization in in- fants younger than 1 year of age: a prospective study in 550 African children. J Neurosurg 103 (6 Suppl):475-481, 2005

47. Warf BC: Congenital idiopathic hydrocephalus of infancy: the results of treatment by endoscopic third ventriculostomy with or without choroid plexus cauterization and suggestions for how it works. Childs Nerv Syst 29:935-940, 2013

48. Warf BC, Bhai S, Kulkarni AV, Mugamba J: Shunt survival after failed endoscopic treatment of hydrocephalus. J Neurosurg Pediatr 10:463-470, 2012

49. Warf BC, Campbell JW: Combined endoscopic third ventriculostomy and choroid plexus cauterization as primary treatment of hydrocephalus for infants with myelomeningocele: long-term results of a prospective intent-to-treat study in 115 East African infants. J Neurosurg Pediatr 2:310-316, 2008

50. Warf BC, Campbell JW, Riddle E: Initial experience with combined endoscopic third ventriculostomy and choroid plexus cauterization for post-hemorrhagic hydrocephalus of prematurity: the importance of prepontine cistern status and the predictive value of FIESTA MRI imaging. Childs Nerv Syst 27:1063-1071, 2011

51. Warf BC, Dewan M, Mugamba J: Management of DandyWalker complex-associated infant hydrocephalus by combined endoscopic third ventriculostomy and choroid plexus cauterization. J Neurosurg Pediatr 8:377-383, 2011

52. Warf BC, Kulkarni AV: Intraoperative assessment of cerebral aqueduct patency and cisternal scarring: impact on success of endoscopic third ventriculostomy in 403 African children. J Neurosurg Pediatr 5:204-209, 2010

53. Warf BC, Mugamba J, Kulkarni AV: Endoscopic third ventriculostomy in the treatment of childhood hydrocephalus in Uganda: report of a scoring system that predicts success. J Neurosurg Pediatr 5:143-148, 2010

54. Warf BC, Ondoma S, Kulkarni A, Donnelly R, Ampeire M, Akona J, et al: Neurocognitive outcome and ventricular volume in children with myelomeningocele treated for hydrocephalus in Uganda. J Neurosurg Pediatr 4:564-570, 2009

55. Warf BC, Tracy S, Mugamba J: Long-term outcome for endoscopic third ventriculostomy alone or in combination with choroid plexus cauterization for congenital aqueductal stenosis in African infants. J Neurosurg Pediatr 10:108-111, 2012

56. Warnke PC, Kreth FW, Ostertag CB: Early postoperative magnetic resonance imaging after resection of malignant glioma: objective evaluation of residual tumor and its influence on regrowth and prognosis. Neurosurgery 36:872-874, 1995

\section{Disclosure}

The authors report no conflict of interest concerning the materials or methods used in this study or the findings specified in this paper.

\section{Author Contributions}

Conception and design: Bhatia, Weil, Ragheb. Acquisition of data: Weil, Fallah. Analysis and interpretation of data: Weil, Fallah. Drafting the article: Weil, Fallah. Critically revising the article: all authors. Reviewed submitted version of manuscript: all authors. Approved the final version of the manuscript on behalf of all authors: Bhatia. Statistical analysis: Fallah. Study supervision: Bhatia, Ragheb.

\section{Correspondence}

Sanjiv Bhatia, Division of Pediatric Neurosurgery, Miami Children's Hospital, Department of Neurosurgery, University of Miami Miller School of Medicine, 3100 SW 62nd Ave., Miami, FL 33155. email: sbhatia@med.miami.edu. 